\title{
ESPECTRO FENOTÍPICO DEL SÍNDROME DE OVARIO POLIQUÍSTICO
}

\author{
Rodrigo Carvajal G. ${ }^{1}$, Cristian Herrera G. ${ }^{2}$, Arnaldo Porcile J. ${ }^{2}$. \\ 1 Programa de Obstetricia y Ginecología, Campus Oriente, Universidad de Chile, Hospital Luis Tisné B., CRS Cordillera \\ Oriente. ${ }^{2}$ Departamento de Obstetricia y Ginecología, Campus Oriente. Universidad de Chile.
}

\section{RESUMEN}

Antecedentes: El síndrome de ovario poliquístico (SOP) es la endocrinopatía más frecuente en la mujer de edad reproductiva, de etiología incierta y presentación clínica heterogénea. Su diagnóstico y definición es aún controversial. La introducción de los criterios del Consenso de Rotterdam generó nuevos fenotipos al incorporar la ecografía transvaginal como elemento diagnóstico, aumentando aún más la heterogeneidad del síndrome. Objetivo: Determinar en una cohorte consecutiva de 102 pacientes con diagnóstico de SOP, su frecuencia, las características clínicas, hormonales y metabólicas, de los cuatro fenotipos posibles según Rotterdam. Resultados: Fenotipo A 62\%, Fenotipo B 21\%, Fenotipo C 9\% y Fenotipo D 8\%. El síndrome metabólico se presentó en $29 \%$ de las pacientes, siendo mayor en los fenotipos A (30\%) y B $(43 \%)$ que en los fenotipos C (11\%) y D (13\%). El $82 \%$ presentaba sobrepeso, siendo significativamente mayor en los fenotipos A ( $88 \%)$ y B $(90 \%)$. Hubo diferencias significativas al comparar las variables puntaje de hirsutismo, SHBG, testosterona total, IAL, volumen ovárico, colesterol total, colesterol HDL, colesterol LDL y glicemia en ayuno. No existieron diferencias significativas entre los grupos al comparar las variables edad, IMC, DHEA-SO4 y triglicéridos. Conclusión: El consenso de Rotterdam agruparía a diferentes fenotipos en un mismo síndrome, que podrían representar distintos grados de severidad de una misma enfermedad. Se desconoce si estos fenotipos poseen los mismos riesgos a largo plazo y sería apresurado tratarlos como una misma entidad.

\section{PALABRAS CLAVE: Síndrome ovario poliquístico, síndrome metabólico, hirsutismo, hiperandroge- nismo, obesidad, riesgo cardiovascular}

\section{SUMMARY}

Background: Policystic ovary syndrome (PCOS) is a very common endocrine disease in women of reproductive age, of uncertain etiology and heterogeneous clinical presentation. The introduction of the Rotterdams Consensus criteria generated new phenotypes by incorporating transvaginal ultrasound, thus increasing the heterogenity of PCOS. Objectives: To determine in a cohort of 102 patients with the diagnosis of PCOS the prevalence, clinical, hormonal and metabolic profile according to Rotterdam. Results: It was determined the Phenotype A $62 \%$ Phenotype B 21\%, Phenotype C 9\% and Phenotype D $8 \%$ of the patients. The metabolic syndrome was present in $29 \%$ of the PCOS patients, being it more frequent in the phenotypes A $(30 \%)$ and B $(43 \%)$ than $C(11 \%)$ and $D(13 \%)$. The $82 \%$ of the patients were overweigth, especially in the phenotypes $A(88 \%)$ and $B(90 \%)$. Also, statistically significant differences were observed when comparing the variables score of hirsutism, free androgen index, total testosterone, HDL cholesterol SHBG, ovarian volume, total cholesterol, glycemia and LDL cholesterol. There were no significant differences between the groups to compare variables such as age, BMI, DHEA and triglycerides. Conclusion: These findings indicate that the 
consensus of Rotterdam would agroup different phenotypes in the same syndrome and that it may represent different degrees of severity of the same disease. Even though, we do not know if these phenotypes posses the same health risks, therefore it is soon to manage the phenotypes as equal entities.

\section{KEY WORDS: Policystic ovary syndrome, metabolic syndrome, hirsutism, hyperandrogenism, obesity, cardiovascular risk}

\section{INTRODUCCIÓN}

El síndrome de ovario poliquístico (SOP) es uno de los desórdenes endocrinos más frecuentes (1), alcanzando una prevalencia entre el 6,5 a $10 \%$ de la población femenina en edad reproductiva $(2,3)$. Se caracteriza por ser un síndrome de disfunción ovárica, asociado a hiperandrogenismo y/o una morfología poliquística del ovario $(4,5)$.

Desde su primera descripción efectuada en 1935 por Stein y Leventhal (6), los criterios para efectuar su diagnóstico han sido modificados en reiteradas oportunidades con el fin de unificar criterios, dado el amplio espectro de mujeres que involucra este síndrome (7,8). Así, en 1990 surgieron los criterios del NIH que incluían en orden de importancia: a) hiperandrogenemia o evidencia clínica de hiperandrogenismo, b) oligoanovulación, y c) exclusión de otros desórdenes endocrinos frecuentemente relacionados, incluyendo hiperprolactinemia, hipotiroidismo y hiperplasia suprarrenal congénita no clásica (7). Posteriormente, en el año 2003, en Rotterdam se incorporan al diagnóstico la presencia de los ovarios poliquísticos a la ecografía transvaginal. De tal manera que, para el diagnóstico se requieren al menos dos de los siguientes elementos: a) oligo o anovulación, b) hiperandrogenismo clínico o hiperandrogenemia y, b) presencia de ovarios poliquísticos en la ecografía transvaginal, además de la exclusión de otras etiologías como la hiperplasia suprarrenal congénita no clásica, tumores productores de andrógenos, síndrome de Cushing y otras formas hiperandrogenismo $(7,8)$.

De esta forma, se extendió y flexibilizó la definición del SOP, incorporándose dos nuevos fenotipos al síndrome: 1) hiperandrogenismo más ovarios poliquísticos y 2) oligoamenorrea más ovarios poliquísticos. Sin embargo, el SOP puede representar un conglomerado de diferentes desórdenes endocrino metabólicos con presentaciones clínicas similares, cuya heterogeneidad se ve aumentada si extendemos el número de fenotipos. De esta manera, surge la necesidad de determinar si los nuevos fenotipos corresponden a la misma enfermedad y si producen los mismos riesgos reproductivos, metabólicos, cardiovasculares y neoplásicos. En efecto, podría tratarse de fenotipos que responden a bases fisiopatológicas diferentes con pronósticos reproductivos y metabólicos desiguales e inciertos, o tratarse de diferentes grados de severidad de una misma enfermedad.

El objetivo del estudio fue determinar en una cohorte de pacientes con diagnóstico de SOP la frecuencia de presentación, las características clínicas, hormonales y metabólicas, de cada uno de los cuatro fenotipos posibles según los criterios de Rotterdam, y evaluar si algún fenotipo difiere del resto en alguna de las variables estudiadas.

\section{PACIENTES Y MÉTODOS}

Análisis retrospectivo de todas las pacientes derivadas a la unidad de ginecología endocrinológica del CRS Cordillera Oriente del Hospital Luis Tisné B., con el diagnóstico presuntivo de SOP, atendidas entre diciembre 2004 y diciembre de 2006. Se seleccionaron las fichas clínicas de aquellas pacientes que cumplían estrictamente los criterios establecidos por el consenso de Rotterdam (8) para SOP (Tabla I). Se excluyeron las pacientes que hayan recibido tratamiento, incluyendo el uso de anticonceptivos hormonales por al menos 3 meses previo a su visita. Los exámenes hormonales fueron realizados en fase folicular temprana al igual que la ecografía transvaginal.

Se diseñó una base de datos con las variables de interés. Se registró edad, peso, índice de masa corporal (IMC), motivo de consulta, estado folicular, puntaje de hirsutismo según Moncada modificado (9), aspecto ecográfico de los ovarios (8), perfil lipídico, testosterona total, DHEA-SO4, SHBG, índice de andrógenos libres (IAL). Asimismo, se buscaron pruebas tiroideas, prolactina y 17-hidroxiprogesterona para descartar otras causas de anovulación crónica e hiperandrogenismo (8). Para descartar causa hipotalámica de anovulación crónica se determinaron niveles de $\mathrm{FSH}$ y $\mathrm{LH}$, principalmente en las pacientes delgadas no hiperandrogénicas.

Se determinó la frecuencia de presentación del síndrome metabólico (SM) de acuerdo a los criterios del American College of Endrocrinology (10), donde se utiliza el IMC en lugar de circunferencia abdominal, por ser el parámetro disponible en nuestro grupo de pacientes. Además, se consignaron los criterios clínicos propios del SM: IMC mayor a 30 $\mathrm{kg} / \mathrm{m} 2$ para diagnosticar obesidad, IMC mayor a 25 
Tabla I

CRITERIOS DIAGNÓSTICOS PARA SOP, SEGÚN EL CONSENSO DE ROTTERDAM, ADOPTADOS POR LA UNIDAD DE GINECOENDOCRINOLOGÍA DEL CRS CORDILLERA ORIENTE

1. Oligoanovulación

2. Signos clínicos o bioquímicos de hiperandrogenismo

3. Ovarios poliquísticos a la ecografía transvaginal
Hiperandrogenismo: escala para hirsutismo $\geq 6$

Hiperandrogenemia: $\mid A L>4,5$

Presencia de 12 o más folículos en cada ovario que midan entre $2-9 \mathrm{~mm}$ de diámetro y/o un volumen ovárico aumentado (>10 ml)

Esta definición no se aplica a mujeres que toman anticonceptivos orales.

Sólo un ovario es suficiente para definir SOP

Si hay evidencia de un folículo dominante $(>10 \mathrm{~mm})$ o un cuerpo lúteo, el examen debe ser repetido en el próximo ciclo.

4. Exclusión de otras patologías hiperandrogénicas y/o anovulantes

SOP: Síndrome de Ovario Poliquístico

$\mathrm{kg} / \mathrm{m} 2$ para sobrepeso, triglicéridos mayor a 150 $\mathrm{mg} / \mathrm{dl}$ para hipertrigliceridemia, HDL bajo $50 \mathrm{mg} / \mathrm{dl}$, presión arterial mayor a 130/85 $\mathrm{mmHg}$, glicemia de ayuno entre $110-126 \mathrm{mg} / \mathrm{dl}$ para intolerancia a la glucosa.

Posteriormente se procedió a agrupar a las pacientes con SOP en uno de los cuatro diferentes fenotipos producto de la aplicación de los criterios de Rotterdam: Fenotipo A (oligoamenorrea + hiperandrogenismo o hiperandrogenemia + ovarios poliquísticos), Fenotipo B (oligoamenorrea + hiperandrogenemia o hiperandrogenismo), Fenotipo C (hiperandrogenismo o hiperandrogenemia + ovarios poliquísticos) y Fenotipo D (oligoamenorrea + ovarios poliquísticos) (Figura 1).

Los resultados son presentados como la media con sus respectivos intervalos de confianza. Se consideró significativo un $p<0,05$. Para la comparación de proporciones se utilizó la prueba de Fisher. Para la comparación de tres o más grupos se utilizó primero la prueba de análisis de varianza, ANOVAKruskal-Wallis de un sentido. Cuando éste fue significativo, se compararon pares de grupos con Dunn's post test. Todos los cálculos estadísticos se realizaron en el programa computacional GRAPH PAD 4.0.

\section{RESULTADOS}

Se registraron 102 pacientes con los criterios de inclusión descritos para SOP entre diciembre 2004 y diciembre de 2006 (Tabla I). Las características de las pacientes se resumen en las Tablas II y III. Edad promedio 27 años (IC95\%: 26-28 años), obesas 57\% o con sobrepeso $82 \%$, e IMC promedio de $31,3 \mathrm{~kg} /$ m2 (IC95\%: 29,9-32,7). Además, hipertensión arterial $12 \%$, hipertrigliceridemia 30\% (promedio $148 \mathrm{mg} / \mathrm{dl}$; IC95\%: 126-170mg/dl), HDL bajo 54\% (promedio 45 $\mathrm{mg} / \mathrm{dl}$; IC95\%: 43-47mg/dl) e intolerancia a la glucosa

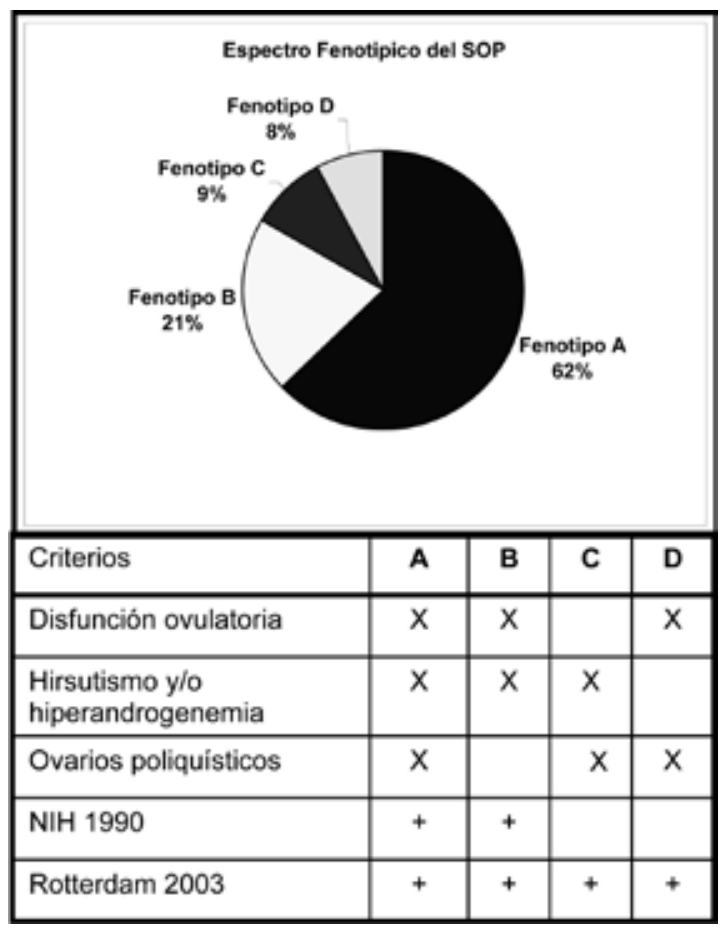

Figura 1. Espectro fenotípico del síndrome de ovario poliquístico según los criterios de Rotterdam. 
en ayuno (promedio $90 \mathrm{mg} / \mathrm{dl}$; IC95\%: $86-93 \mathrm{mg} / \mathrm{dl}$ ) o post carga de glucosa en $15 \%$ (Tabla II). La prevalencia de SM en este grupo de pacientes con SOP es de 29\% (Tabla II y Figura 2). Así mismo, las pacientes con SOP son hiperandrogénicas, clínicamente manifestado por hirsutismo (puntaje de hirsutismo 7,2; IC95\%: 6,4-8,0) y bioquímicamente por IAL aumentado (13,6; IC95\%: 10,98-16,29) (Tabla III).

Se descartó otras causa de anovulación crónica e hiperandrogenismo en las pacientes estudiadas con niveles normales de $17-\mathrm{OH}$-progesterona $(0,91$ $\mathrm{ng} / \mathrm{ml}$; IC95\%: 0,06-1,88 ng/ml), prolactina (15 ng/ $\mathrm{ml}$; IC95\%: $12-18 \mathrm{ng} / \mathrm{ml})$, TSH (2,48 mUl/ml; IC95\%: 2,1-2,8 mUI/L), DHEA-SO4 (280 ug/dl; IC95\%: 196$364 \mathrm{ug} / \mathrm{dl})$, LH (15,5 UI/L; IC95\%: 11,6-19 UI/L) y FSH (4,66 UI/L; IC95\%: 4,2-5,0 UI/L) (Tabla III).

Al agruparlos en los cuatro diferentes fenotipos se distribuyeron por frecuencia en orden decreciente: fenotipo A $62 \%$, fenotipo B $21 \%$, fenotipo C $9 \%$ y el fenotipo $D 8 \%$ (Figura 1). Los fenotipos $A+B$, que cumplen los antiguos criterios del NIH 1990, suman el $83 \%$ del total de pacientes con SOP. El $21 \%$ del total de pacientes no presentaron ovarios poliquístico a la ecografía transvaginal, sólo el $8 \%$ no presentó hiperandrogenismo ni hiperandrogenemia (fenotipo D).

El análisis comparativo entre los diferentes fenotipos se resume en las Tablas IV y V. Se observa por definición de los propios fenotipos, diferencias entre los puntajes de hirsutismo, IAL, testosterona total y volumen ovárico (Tabla IV y Figura $3 \mathrm{~A}, \mathrm{~B}, \mathrm{C}$, I). El volumen ovárico fue menor para el fenotipo $B$ (6,3 ml, IC95\%: 5,7-6,9) con respecto a los otros fenotipos y principalmente al fenotipo A (Figura 3C).

La frecuencia de pacientes que presentaron $\mathrm{SM}$ en cada fenotipo fue mayor en el fenotipo $B(43 \%)$ y fenotipo $\mathrm{A}(30 \%)$, que cumplen con los antiguos criterios $\mathrm{NIH}$ para SOP que los nuevos fenotipos Rotterdam C (11\%) y D (13\%) (Tabla V y Figura 2). La proporción de obesidad, sobrepeso, HTA, hipertrigliceridemia, HDL bajo

Tabla II

\section{CARACTERÍSTICAS DE LAS PACIENTES CON} SÍNDROME DE OVARIO POLIQUÍSTICO

\begin{tabular}{lc}
\hline Variables & $\mathrm{n}(\%)$ \\
\hline Síndrome metabólico & $30(29,4)$ \\
Obesidad & $58(56,9)$ \\
Sobrepeso & $84(82,3)$ \\
Hipertensión arterial & $12(11,7)$ \\
Hipertrigliceridemia & $31(30,4)$ \\
HDL bajo & $55(53,9)$ \\
Intolerancia a la glucosa (ayuno) & $15(14,7)$ \\
\hline
\end{tabular}

Tabla III

CARACTERÍSTICAS CLÍNICAS Y DE LABORATORIO DE LAS PACIENTES CON SÍNDROME DE OVARIO POLIQUÍSTICO (n: 102)

\begin{tabular}{ll}
\hline Variable & Promedio (IC95\%) \\
\hline Edad (años) & $27,3(26,0-28,5)$ \\
IMC (kg/m2) & $31,3(29,9-32,7)$ \\
Puntaje hirsutismo & $7,2(6,4-8)$ \\
Índice andrógenos libres & $13,6(10,9-16,3)$ \\
SHBG (nmol/L) & $32,1(28-36)$ \\
Volumen ovárico (ml) & $9,1(8,6-9,6)$ \\
Testosterona total (nmol/L) & $3,0(2,7-3,3)$ \\
DHEA-SO4 (ug/dl) & $280,2(196,5-364)$ \\
LH (Ul/L) & $15,6(11,6-19,5)$ \\
FSH (Ul/L) & $4,7(4,2-5)$ \\
Colesterol (mg/dl) & $173,6(166,3-180,9)$ \\
HDL (mg/dl) & $45,4(43,1-47,6)$ \\
LDL (mg/dl) & $103,3(95,5-111)$ \\
TGL (mg/dl) & $148,8(126,9-170,7)$ \\
Glicemia (mg/dl) & $90,2(86,7-93,6)$ \\
17OH progesterona (ng/ml) & $0,91([-0,06]-1,8)$ \\
Prolactina (ng/ml) & $15,8(12,9-18,5)$ \\
TSH (mUl/L) & $2,5(2,1-2,8)$ \\
\hline
\end{tabular}

Sindrome Metabólico en SOP

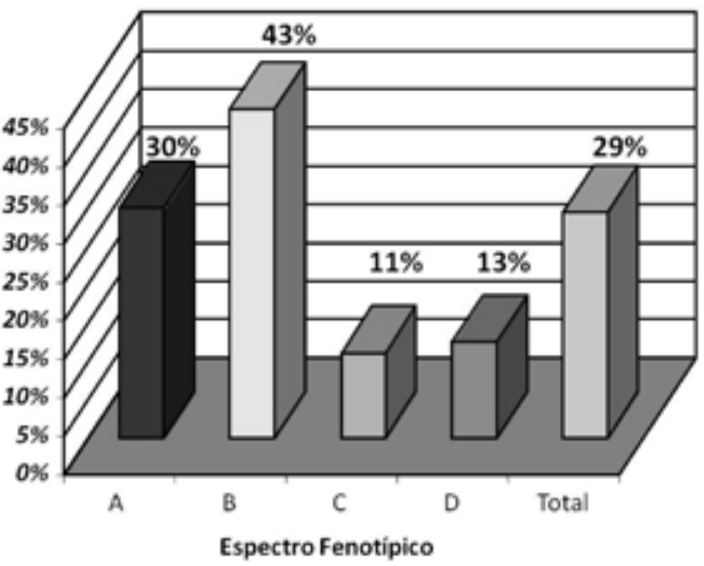

Figura 2. Prevalencia de Síndrome Metabólico en pacientes con Síndrome de Ovario Poliquístico. 
e intolerancia a la glucosa en ayuno, fue en general mayores en los fenotipos $A$ y $B$ que los fenotipos $C$ y $D$ (Tabla V). Sólo hubo diferencias significas al comparar la proporción de sobrepeso entre los fenotipos A (88\% de sobrepeso) y $C$ ( $44 \%$ de sobrepeso) $(p=0,0071)$ y entre el fenotipo B ( $90 \%$ de sobrepeso) y el fenotipo $C$ $(p=0,0139)$ (Tabla $V)$. Igualmente, se encontraron diferencias al comparar el porcentaje de pacientes que presentaban HDL bajo entre los fenotipos A (56\%) y D (13\%) y entre los fenotipos B (67\%) y D (Tabla V).

Hubo diferencias significativas al comparar las medias de las variables SHBG, colesterol total, colesterol HDL, colesterol LDL y glicemia de ayuno (Tabla IV y Figura 3). En efecto, SHBG es mayor en el fenotipo D (78 nmol/l, IC95\%: 15-141) con respecto a los otros tres fenotipos (Figura 3D). Con respecto al colesterol total, el fenotipo D $(205 \mathrm{mg} / \mathrm{dl}$, IC95\%: 173-237) es mayor que el fenotipo C (150 $\mathrm{mg} / \mathrm{dl}, \mathrm{IC} 95 \%$ : 121-179), ocurriendo lo mismo para el colesterol LDL, fenotipo D $139 \mathrm{mg} / \mathrm{dl}$ (IC95\%: 105-172) vs $80 \mathrm{mg} / \mathrm{dl}$ (IC95\%: 49-111) para el fenotipo C (Figura 3E y 3F). El colesterol HDL fue significativamente mayor en el fenotipo $D$ que en el fenotipo A (Tabla IV y Figura $3 \mathrm{H}$ ). La glicemia de ayuno, fue mayor en el fenotipo B $95,3 \mathrm{mg} / \mathrm{dl}$ (IC95\%: 90,1-100,5) vs el fenotipo A 83,19 mg/dl (IC95\%: 83,45-88,92) (Tabla IV y Figura 3G).
Tabla V

CARACTERÍSTICAS DE LOS DIFERENTES FENOTIPOS DE SÍNDROME DE OVARIO POLIQUÍSTICO

\begin{tabular}{lcccc}
\hline & \multicolumn{4}{c}{ Fenotipos n(\%) } \\
& A & B & C & D \\
\hline Frecuencia & $64(63)$ & $21(20,5)$ & $9(8,8)$ & $8(7,8)$ \\
$\begin{array}{l}\text { Síndrome } \\
\text { metabólico }\end{array}$ & $19(30)$ & $9(43)$ & $1(11)$ & $1(13)$ \\
Obesidad & $39(61)$ & $13(62)$ & $3(33)$ & $3(38)$ \\
Sobrepeso & $56(88)^{\text {a }}$ & $19(90)^{\text {b }}$ & $4(44)$ a,b & $5(63)$ \\
Hipertensión & & & & \\
arterial & $6(9)$ & $6(28)$ & -- & -- \\
Hipertrigliceridemia & $17(27)$ & $10(48)$ & $1(11)$ & $3(38)$ \\
HDL bajo & $36(56)^{c}$ & $14(67){ }^{d}$ & $4(44)$ & $1(13)$ \\
ITG ayuno & $9(14)$ & $4(19)$ & $1(11)$ & $1(13)$ \\
\hline
\end{tabular}

ITG: intolerancia a la glucosa ayuno. a: $p=0,0071$; $b$ : $p=0,0139$; $: p=0,0056 ; d: p=0,014$. El resto de los valores presentaron un $p>0,05$ por lo que no se detallan (Test de Fisher).

Tabla IV

\section{COMPARACIÓN DE PARÁMETROS CLÍNICOS Y DE LABORATORIOS ENTRE LOS DIFERENTES FENOTIPOS DEL SOP SEGÚN CRITERIOS DE ROTTERDAM}

\begin{tabular}{|c|c|c|c|c|}
\hline Variables & Fenotipo A & Fenotipo B & Fenotipo C & Fenotipo D \\
\hline n (\%) & $64(62,7 \%)$ & $21(20,5 \%)$ & $9(8,8 \%)$ & $8(7,8 \%)$ \\
\hline Edad (años) & $26,3(24,8-27,8)$ & $30,2(27,2-33,1)$ & $26,5(20,4-32,6)$ & $28,6(23,9-33,2)$ \\
\hline IMC (kg/m2) & $31,6(30,2-33)$ & $33,3(29,3-37,3)$ & $28,4(20,4-36,4)$ & $27,9(22,1-33,7)$ \\
\hline Puntaje hirsutismo $^{a}$ & $8(6,5-8,5)$ & $7,5(5,8-9,6)$ & $8,0(5,2-9,7)$ & $4(0,3-5,9)$ \\
\hline $\mathrm{IAL}^{\mathrm{a}, \mathrm{b}}$ & $25,1(9,9-40,1)$ & $13,0(6,6-19,3)$ & $13,3(0,3-27,0)$ & $3,1(2,1-4,1)$ \\
\hline SHBG (nmol/L) $)^{a, b, c}$ & $29,9(24,7-35,2)$ & $27,2(21,9-32,6)$ & $40,6(24,1-57,1)$ & $78,1(15,0-141,2)$ \\
\hline DHEA-SO4 (ug/dl) & $211(178,1-243,8)$ & $228,3(161,3-295,4)$ & $243(183,4-302,6)$ & $187,1(2,7-377,0)$ \\
\hline Testosterona total $(\mathrm{nmol} / \mathrm{L})^{a}$ & $3,2(2,9-3,6)$ & $2,5(2,1-3,0)$ & $3,3(1,1-5,5)$ & $1,9(1,2-2,7)$ \\
\hline LH/FSH & 3,8 & 3,9 & 0,5 & 3,3 \\
\hline Colesterol $(\mathrm{mg} / \mathrm{dl})^{\mathrm{c}}$ & $170,1(160,7-179,4)$ & $176,3(161,1-195,5)$ & $150,7(121,9-179,4)$ & $205,5(173,6-237,4)$ \\
\hline $\mathrm{HDL}(\mathrm{mg} / \mathrm{dl})^{\mathrm{a}}$ & $43,3(40,9-45,7)$ & $44,5(39,7-49,2)$ & $47,8(35,5-60,1)$ & $60,8(46,2-75,3)$ \\
\hline $\operatorname{LDL}(\mathrm{mg} / \mathrm{dl})^{\mathrm{c}}$ & $99,8(91,2-108,4)$ & $110,3(89,1-131,4)$ & $80,7(49,6-111,9)$ & $139,2(105,9-172,6)$ \\
\hline TGL (mg/dl) & $135,8(115,4-156,2)$ & $179,6(115,9-243,3)$ & $110,3(54,4-166,2)$ & $128,8(70,2-187,3)$ \\
\hline Volumen ovárico $(\mathrm{ml})^{\mathrm{d}}$ & $10,1(9,4-10,8)$ & $6,3(5,7-6,9)$ & $8,9(7,9-9,8)$ & $8,1(6,4-9,8)$ \\
\hline Glicemia (mg/dl) ${ }^{d}$ & $86,1(83,4-88,9)$ & $95,3(90,1-100,5)$ & $90,6(79,1-102,3)$ & $87,4(79,7-95,1)$ \\
\hline
\end{tabular}

${ }^{a}$ Fenotipo A significativamente diferente del fenotipo $D(p<0,05) .{ }^{b}$ Fenotipo $B$ significativamente diferente del fenotipo $D(p<0,05) .{ }^{C}$ Fenotipo $C$ significativamente diferente del fenotipo $D(p<005) .{ }^{d}$ Fenotipo $A$ significativamente diferente del fenotipo $B(p<0,05)$. 


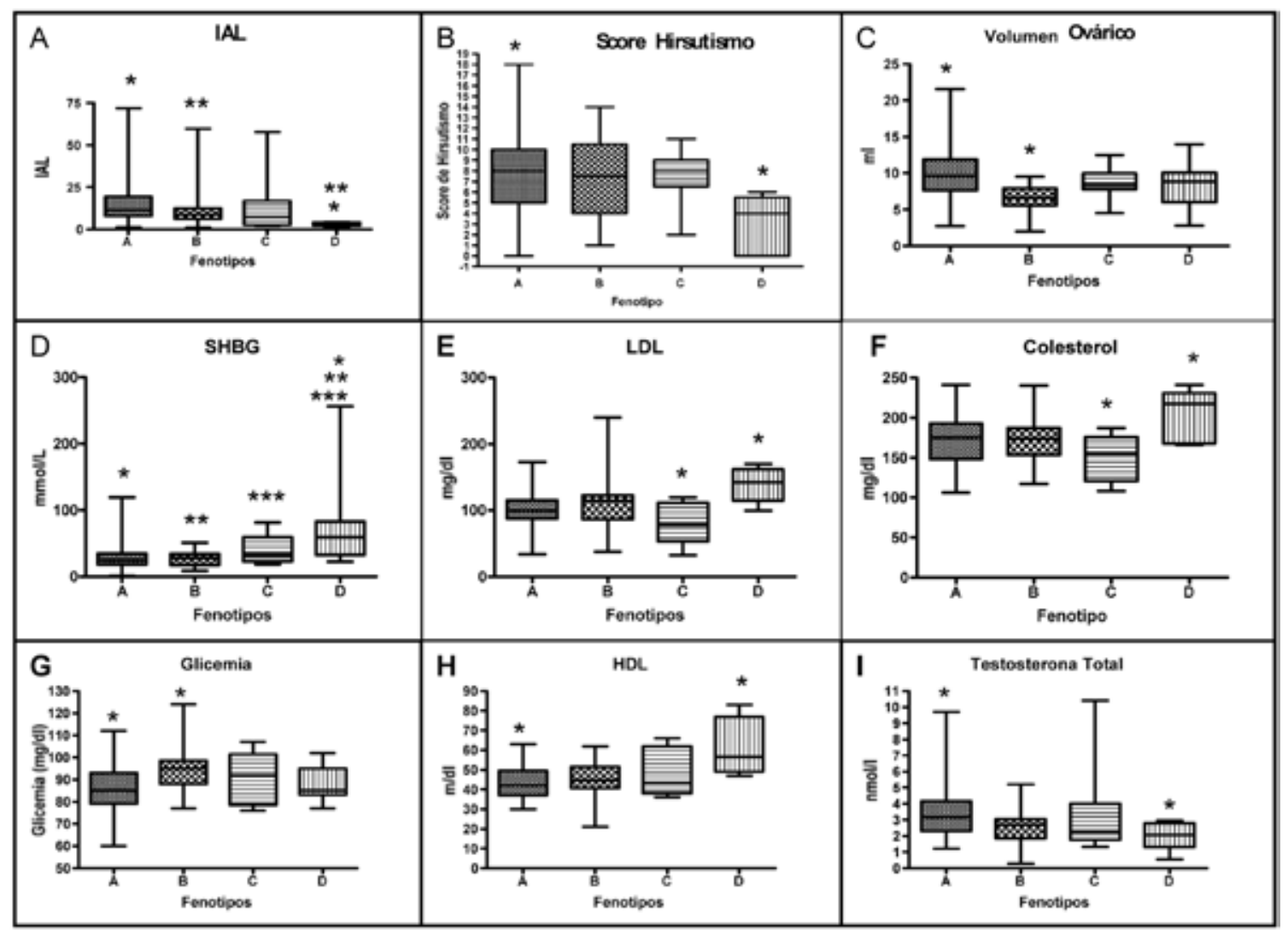

Figura 3. Análisis estadístico entre los diferentes fenotipos de síndrome de ovario poliquístico $(p<0,05$ entre ${ }^{*} y^{*} ; p<0,05$ entre ${ }^{* *} y^{* *} ; p<0,05$ entre $\left.{ }^{* *} y^{* * *}\right)$.

No hubo diferencias significativas entre los diferentes grupos al comparar las variables edad, IMC, DHEAS-SO4 y triglicéridos (Tabla IV). Sin embargo, existe una tendencia no significativa del fenotipo D, hacia un menor IMC $(27 \mathrm{mg} / \mathrm{kg} 2$, IC95\%: $22-$ 33), menor DHEA-SO4 (187 ug/dl, IC95\%: 2,3-377 $\mathrm{ug} / \mathrm{dl}$ ) que el resto de los grupos (Tabla IV).

\section{DISCUSIÓN}

En mayo del 2003, un grupo de expertos se reunió en Rotterdam con el objetivo de evaluar los avances en el entendimiento del SOP y, de ser necesario, redefinir los criterios diagnósticos bajo la luz de estos nuevos conocimientos (8). Si bien se llegó a un consenso, no se lograron resolver ciertas controversias, como justificar la incorporación de los dos nuevos fenotipos al síndrome: las pacientes ovulatorias hiperandrogénicas con ovarios poliquísticos y las normoandrogénicas con ovarios poliquísticos y oligoamenorrea $(11,12,13,14)$.

EI SOP por su naturaleza es una concurrencia de signos, síntomas y alteraciones de laboratorio que pueden combinarse de varias modos $(11,12,13,14)$.
Como su etiología no es clara, no existe un "gold standard" de referencia, y se reconocen bases genéticas y medio ambientales que pueden influir, es que se recurre a criterios establecidos por expertos que se van modificando en la medida que evoluciona el conocimiento sobre el tema.

Nuestro grupo de pacientes con SOP, en concordancia a lo descrito internacionalmente, son pacientes jóvenes, en su gran mayoría obesas o con sobrepeso, hiperandrogénicas e hiperandrogenémicas y normogonadotrópicas. Además, si notamos su asociación a dislipidemia e intolerancia a la glucosa nos damos cuenta que son pacientes con elevado riesgo cardiovascular y de desarrollar diabetes mellitus a edades tempranas, por lo cual las intervenciones precoces se vuelven aún más relevantes no solamente pensando en su fertilidad (Tabla II y III). Sin embargo, el impacto real aún se desconoce por la heterogeneidad del síndrome y la falta de seguimiento a largo plazo para determinar el impacto en cada fenotipo, de ahí la importancia de este estudio en una muestra de pacientes chilenas.

Carmina y cols (15), estudiaron pacientes con ovarios poliquísticos a la ecografía transvaginal y 
signos clínicos y/o bioquímicos de hiperandrogenismo, asociados a ciclos ovulatorios normales, observando que este grupo de pacientes presentaba niveles mayores de andrógenos circulantes e hiperinsulinemia, además mayores niveles de 17-OHprogesterona en respuesta a la acción de análogos de $\mathrm{GnRH}$ comparados con controles no SOP. Sin embargo, los niveles de hiperinsulinismo e hiperandrogenemia eran significativamente menores que aquellas pacientes SOP según los criterios del NIH. Por otro lado, se desconoce si este grupo de individuos posee los mismos riesgos cardiovasculares y reproductivos que las pacientes SOP según los criterios del NIH (7). En efecto, en un estudio retrospectivo cuyo objetivo eran determinar el riesgo relativo del SM en pacientes con SOP según los criterios de Rotterdam, se encontró que el fenotipo no androgénico de ovarios poliquísticos y oligoamneorrea (fenotipo D) presentaba el mismo riesgo que las pacientes controles sanas no SOP, incluso en las mujeres obesas (16). De esta forma, frente a la controversia de aumentar la heterogeneidad del SOP y con la débil evidencia disponible sobre los nuevos fenotipos, algunos grupos consideran muy prematuro adoptar los nuevos criterios de Rotterdam, ya que podrían significar efectos negativos a nivel de la práctica clínica, investigación científica y calidad de vida de las pacientes (14).

En el cohorte de pacientes con SOP analizadas, el $83 \%$ cumplía los criterios del NIH (fenotipos $A+B)$, es decir eran hiperandrogénicas y/o hiperandrogenémicas, además de oligoanovulatorias. Al incorporar el criterio ecográfico de ovarios poliquísticos, se incorporan dos nuevos fenotipos, C y D, aumentando el número de pacientes con el síndrome en un $20 \%$ (Figura 1).

Del total de pacientes analizadas, el $79 \%$ presentaban ovarios poliquísticos a la ecografía transvaginal (fenotipos $A+C+D$ ), pudiendo corresponder el $21 \%$ restante (fenotipo $B$ ) a falsos negativos ecográficos, ya que este fenotipo está ya presente en los antiguos criterios del NIH (7). De este modo, pudiera plantearse que la incorporación de la ecografía transvaginal como criterio diagnóstico aumenta la sensibilidad pero a la vez disminuye la especificidad en la pesquisa del $\operatorname{SOP}(12,13,14)$. Desde este punto de vista, surge la interrogante sobre la validez de los dos nuevos fenotipos incorporados al síndrome, ovarios poliquísticos con oligoamenorrea y ovarios poliquísticos con hiperandrogenismo (fenotipos D y C). No está claro aún que estos últimos fenotipos, no así con los fenotipos NIH, posean igualmente mal pronóstico reproductivo o representen factores de riesgo cardiovascular a largo plazo, sobretodo con la ausencia de hiperandrogenismo $(13,14,15,16)$.
Al evaluar cada fenotipo por separado encontramos que el fenotipo $A$, el denominado fenotipo SOP completo, es el más frecuente de todos con un $62 \%$. Sin embargo, podría plantearse que por ser un fenotipo de características tan floridas y llamativas es diagnosticado más fácilmente $\mathrm{y}$, por lo tanto, con mayor frecuencia $(11,17)$. Según nuestros resultados y los de la literatura tienden a ser pacientes más jóvenes, obesas, con mayores puntajes de hirsutismo e índices de andrógenos libres, menores niveles de SHBG y mayores volúmenes ováricos que el resto de los fenotipos (17) (Tabla IV y Figura 3). Lo anterior, hace suponer un grado mayor de severidad del síndrome y, tal vez, aunque no se haya medido directamente, mayores niveles de insulino resistencia, inferido por los bajos niveles de SHBG, la obesidad y los bajos niveles de HDL (13).

El fenotipo B, oligoameorrea e hiperandrogenismo, incluido en los antiguos criterios del $\mathrm{NIH}$ es el segundo más frecuente, con un $21 \%$ del total de pacientes con SOP. Tienden a ser, sin lograr significancia estadística, las pacientes de mayor edad, obesidad e hirsutismo, además, por definición poseen ovarios normales a la ecografía transvaginal.

El nuevo fenotipo ovulatorio del SOP según Rotterdam, fenotipo $\mathrm{C}$, no difiere significativamente de los antiguos fenotipos SOP, fenotipo A y B. En cambio, si posee diferencias estadísticamente significativas con el nuevo fenotipo no androgénico del SOP, fenotipo D, en los niveles de SHBG, colesterol total y LDL. Estos resultados son concordantes con lo reportado en la literatura que demuestran que el fenotipo SOP ovulatorio posee alteraciones en los niveles de gonadotrofinas circulantes, así como, mayores niveles de insulina circulantes, justificando su agrupación junto a los otros fenotipos $\operatorname{SOP}(17,18)$.

Sólo el $8 \%$ del total de pacientes pertenece al nuevo fenotipo no androgénico del SOP o fenotipo $D$, ya que no presentan hiperandrogenismo clínico ni de laboratorio. De esta forma, el fenotipo de oligoanovulación más ovarios poliquísticos a la ecografía transvaginal es el más controversial de todos, ya que no cumple con lo fundamental de la descripción inicial de Stein y Leventhal (6), que es un hiperandrogenismo de origen ovárico. Sin embargo, según nuestros resultados, se encontraron niveles mayores de SHBG, colesterol total, colesterol LDL, colesterol HDL pudiendo con esto justificar su incorporación al síndrome. En efecto, el fenotipo D presenta una disfunción ovulatoria (oligoanovulación y ovarios poliquísticos) con una disfunción metabólica (dislipidemia), que representan a su vez factores de riesgo cardiovascular y un mal pronóstico reproductivo, característicos de las pacientes con SOP. Así, 
los cuatro fenotipos podrían interpretarse como distintos grados de severidad de una misma disfunción metabólica, siendo el fenotipo D el más leve de ellos, por presentar una tendencia hacia menores IMC, IAL, puntajes de hirsutismo, testosterona total, glicemia y mayores niveles de HDL (Tabla IV y Figura 3).

Un aspecto sustancial es establecer si los cuatros fenotipos del SOP, ya sea porque son manifestaciones heterogéneas de una misma patología o, en el extremo contrario, entidades diferentes que se agrupan por compartir algunas características, conllevan los mismo riesgos cardiovasculares y reproductivos $(8,19)$. En efecto, es sabido que el SOP representa un factor de riesgo para abortos espontáneos, abortos recurrentes, restricción del crecimiento intrauterino (RCIU), diabetes gestacional y preeclampsia, pero no se sabe si ocurren en igual medida con cada uno de estos nuevos fenotipos $(8,19,20)$. Del mismo modo, el SOP representa un importante factor de riesgo cardiovascular, con mayor prevalencia de SM, insulino resistencia y diabetes mellitus, desconociéndose si todos los fenotipos son igualmente riesgosos (16).

Nuestra cohorte de pacientes con SOP presenta SM en el $29 \%$ de ellas. Sin embargo, como utilizamos el IMC, en lugar de la circunferencia abdominal, podríamos estar sobreestimando la frecuencia de este síndrome si lo comparamos con los estudios que utilizan los criterios del ATP III (28). A pesar de que pareciera ser más frecuente en los fenotipos $\mathrm{NIH}$, no logramos demostrar diferencias estadísticamente significativas en la proporción de SM. Sin embargo, encontramos diferencias significativas en la proporción de sobrepeso y HDL bajo, que fueron mayores en los fenotipos $A$ y $B$ en comparación con los fenotipos C y D. Lo anterior, en alguna medida nos indica que pudiera existir un grado mayor de resistencia a la insulina en los fenotipos SOP NIH, pero que no fuimos capaces de demostrarlo en el porcentaje de presentación de SM por nuestro pequeño tamaño muestral, y por no disponer de elementos diagnósticos más directos de resistencia a la insulina como insulinemia basal y poscarga.

Aun así, se describe la asociación del SOP con resistencia a la insulina de hasta un $60 \%$, por lo tanto, consideramos de importancia esclarecer si existen diferencias en los niveles de insulinemia e insulino resistencia entre estos grupos, que pudiesen explicar el fenotipo y sobre todo por su impacto cardiovascular y enfrentamiento terapéutico $(21,22,23)$. Estudios demuestran que niveles bajos de SHBG, se reflejan en un aumento en la biodisponibilidad de testosterona libre (24), y que la insulina regula negativamente la producción hepática de SHBG $(24,25)$. Más aún, mujeres con mayor cantidad de masa grasa a nivel abdominal, tienen menores niveles de SHBG y alta producción de testosterona y dihidrotestosterona, comparada con aquellas mujeres con obesidad de distribución corporal periférica (26), por lo que consideramos que la medida de la circunferencia abdominal es una práctica que se debería adquirir en el manejo de este tipo de pacientes. En efecto, los niveles de andrógenos se elevan en conjunto con los de insulinemia y se ha reportado que la insulina es capaz de estimular directamente la producción de andrógenos ováricos (27). Sin embargo, la hiperinsulinemia no se correlaciona en forma proporcional con los puntajes de hirsutismo. Tal condición, podría deberse a que el crecimiento del pelo terminal no sólo se produzca por la hiperandrogenemia sino también por la hiperinsulinemia.

Destacamos como sesgos de selección en nuestra evaluación que estudiamos pacientes seleccionadas previamente por los centros de derivación y el motivo de consulta de las pacientes, ya que nuestro grupo corresponde a una unidad de endocrinología ginecológica y no de infertilidad. En el mismo sentido, para realmente esclarecer si el espectro fenotípico de nuestro grupo de pacientes con SOP constituyen matices de una misma enfermedad, faltaría un grupo control de pacientes sanas. Tal situación, sería posible con un grupo de pacientes que consulte con infertilidad por factor masculino, circunstancia que escapa de nuestra unidad pero que, aún así, es una tarea pendiente por dilucidar.

EI SOP es una de las endocrinopatías más frecuente en la mujer, existiendo aún muchas interrogantes acerca del alcance real que éste tiene. Desde este punto de vista, el consenso de Rotterdam es hoy en día una herramienta de gran ayuda para unificar criterios diagnósticos, haciendo posible la comparación entre diferentes estudios clínicos, teniendo siempre la precaución y entendimiento de que el SOP es un síndrome, y por consecuencia puede reunir varias entidades o enfermedades que aún no han podido individualizarse.

Los resultados expuestos no nos permiten asegurar que existan diferencias sustanciales entre las características clínicas y de laboratorio de los grupos analizados, aparte de las diferencias establecidas para definir cada fenotipo. De esta manera, el consenso de Rotterdam estaría agrupando efectivamente a diferentes fenotipos de un mismo síndrome. Puede plantearse que los diferentes fenotipos representan distintos grados de severidad de una misma enfermedad, cuya base fisiopatológica aún no ha logrado identificarse, pero que la insulino resistencia es una fuerte candidata (21-26).

\section{CONCLUSIÓN}

El consenso de Rotterdam estaría agrupando a diferentes fenotipos en un mismo síndrome que 
podrían representar distintos grados de severidad de una misma enfermedad. Se desconoce si estos fenotipos poseen los mismos riesgos a largo plazo y por lo tanto es apresurado aún tratar los diferentes fenotipos como una misma entidad. Como desafío para futuros estudios queda por determinar el riesgo relativo específico sobre el pronóstico reproductivo, cardiovascular y neoplásico de cada uno de estos nuevos fenotipos del SOP, según los criterios de Rotterdam, como única forma de determinar si es correcto o no agruparlos y manejarlos bajo un mismo síndrome.

\section{BIBLIOGRAFÍA}

1. Azziz R,Woods KS,Reyna R, Key TJ, Knochenhauer $\mathrm{ES}$, Yildiz BO. The prevalence and features of the polycistic ovary syndrome in an unselected population.J Clin Endocrinol Metab 2004;89:2745-9.

2. Knochenhauer ES, Key TJ, Kahsar-Miller M, Waggoner W, Boots LR, Azziz R. Prevalence of the polycystic ovary syndrome in unselected black and white women of the southeastern United States: a prospective study. J Clin Endocrinol Metab 1998;83:3078-82.

3. Goodarzi M, Azziz R. Diagnosis, epidemiology and genetics of the polycyistic ovary syndrome. Best Pract Res Clin Endocrinol Metab 2006;20:193-205.

4. Laven JS, Imani B, Eijkemans MJ, Fauser BC. New approaches to PCOS and other forms of anovulation. Obstet Gynecol Surv 2002;57:755-67.

5. Carmina E, Rosato $F$, Jannì A, Rizzo M, Longo RA. Relative prevalence of different androgen excess disorders in 950 women referred because of clinical hyperandrogenism. J Clin Endocrinol Metab 2006;91:2-6.

6. Stein IF, Leventhal ML. Amenorrhea associated with bilateral policystics ovaries. Am J Obstet Gynecol 1935;29:181-91.

7. Zawadski JK, Dunaif A. Diagnostic criteria for polycystic ovary syndrome: towards a rational approach. In: Dunaif A, Givens JR, Haseltine F, eds. Polycystic ovary syndrome. Boston: Blackwell Scientific, 1992:377-84.

8. Rotterdam ESHRE/ASRM-Sponsored PCOS Consensus Workshop Group. Revised 2003 consensus on diagnostic criteria and long-term health risks related to polycystic ovary syndrome. Fertil Steril 2003;81:19-25.

9. Moncada E. Familial study of hirsutism. J Clin Endocrinol Metab 1970;31:556-64.

10. Einhorn D, Reaven GM, Cobin RH, Ford E, Ganda $\mathrm{OP}$, et al. American College of Endocrinology position statement on the insulin resistance syndrome. Endocr Pract 2003;9:237-52.

11. Carmina E. The spectrum of androgen excess disorders. Fertil Steril 2006;85:1582-5.

12. Chang WY, Knochenhauer ES, Bartolucci AA, Azziz R. Phenotypic spectrum of polycystic ovary syndrome: clinical and biochemical characterization of the three major clinical subgroups. Fertil Steril 2005;83:1717-23.

13. Franks S. Controversy in clinical endocrinology: diagnosis of polycystic ovarian syndrome: in defense of the Rotterdam criteria. J Clinical Endocrinol Metab 2006,91:786-9.
14. Azziz R. Controversy in clinical endocrinology: diagnosis of polycystic ovarian syndrome: the Rotterdam criteria are premature. J Clinical Endocrinol Metab 2006;91:781-5.

15. Carmina E, Chu MC, Longo RA, Rini GB, Lobo RA. Phenotypic variation in hyperandrogenic women influences the findings of abnormal metabolic and cardiovascular risk parameters. J Clin Endocrinol Metab 2005;90:2545-9.

16. Shroff R, Syrop CH, Davis W, Van Voorhis BJ, Dokras A. Risk of metabolic complications in the new PCOS phenotypes based on the Rotterdam criteria. Fertil Steril 2007;88:1389-95.

17. Dewailly D, Catteau-Jonard S, Reyss A, Leroy M, Pigny P.Oligoanovulation with polycystic ovaries but not overt hyperandrogenism. J Clin Endocrinol Metab 2006;91:3922-7.

18. Barber TM, Wass JA, McCarthy MI, Franks S. Metabolic characteristics of women with polycystic ovaries and oligo-amenorrhoea but normal androgen levels: implications for the management of polycystic ovary syndrome. Clin Endocrinol 2007;66.513-7.

19. Grundy SM, Cleeman JI, Daniels SR, Daniels SR, Donato $\mathrm{KA}$, Eckel RH, et al; American Heart Association; National Heart, Lung, and Blood Institute. Diagnosis and management of the metabolic syndrome: an American Heart Association/National Heart, Lung, and Blood Institute Scientific Statement. Circulation 2005; 112: 2735-52.

20. Homburg R. Pregnancy complications in PCOS. Best Pract Res Clin Endocrinol Metab 2006;20:281-92.

21. Dunaif $A$. Insulin resistance and the polycyctic ovarian syndrome: mechanism and implications for pathogenesis. Endoc Rev 1997;18:774-800.

22. Legro RS, Castracane VD and Kauffman RP. Detecting insulin resistance in polycystic ovary syndrome: purposes and pitfalls. Obstet Gynecol Surv 2004;59:141-54.

23. Baillargeon JP, Nestler JE. Commentary: polycystyc ovary syndrome: a syndrome of ovarian hypersensivity to insulin? J Clin Endocrinol Metab 2006;91:22-4.

24. Ehrmann D. Polycystic ovary syndrome. N Engl J Med 2005;352:1223-36.

25. Yen S. Anovulación crónica hiperandrogénica. Endocrinología de la reproducción. Cuarta Edición. Bs. Aires, Bogotá, Caracas, Ciudad de México, Sao Paulo. Editorial Panamericana, 2001:465-510.

26. Kirchengast S, Huber J (2001) Body composition characteristics and body fat distribution in lean women with polycystic ovary syndrome. Hum Reprod 2001;16:1255-60.

27. Nestler J, Jakubowicz D, Falcon de Vargas A, Brik C, Quintero N, Medina F. Insulin stimulates testosterone biosynthesis by human thecal cells from women with polycystic ovary syndrome by activating its own receptor and using inositolglycan mediators as the signal transduction system. J Clin Endocrinol Metab 1998;83:2001-5.

28. National Cholesterol Education Program (NCEP) Expert Panel on Detection, Evaluation, and Treatment of High Blood Cholesterol in Adults (Adult Treatment Panel III). Third report of the national cholesterol education program (NCEP) expert panel on detection, evaluation, and treatment of high blood cholesterol in adults (Adult Treatment Panel III). Final report. Circulation 2002;106:3143-421. 